\title{
Study on the Speech Processing Strategies of Bi-sentences of Thai Native Speakers
}

\author{
Wang Ruifeng \\ Beijing Language and Culture University \\ Beijing, China
}

\begin{abstract}
By analyzing interlanguage corpus and sentence acquisition stages of 9 patterns of Chinese Bi-sentences of intermediate Thai native speakers, this paper explored the speech processing strategies of $\mathrm{Bi}$-sentences. It is found that the speech processing strategies of Thai native speakers experienced the development process from the word order strategy for single typical Bi-sentences to the word order strategy for multiconditional Bi-sentences. The speech processing strategies of inner-sentence semantic components experienced the development process from the strategy of the single typical word combination to the strategy of multi-conditional word combination. The speech processing strategies of inner-sentence grammatical components underwent the development process from the strategy of skipping grammatical components to the strategy of using grammatical components.
\end{abstract}

Keywords—second language acquisition; the speech processing strategies of Bi-sentences; Thai native speakers

\section{INTRODUCTION}

The study on the Multidimensional Model of western second language acquisition found that learners could adopt corresponding language cognitive processing strategies in different stages during second language acquisition. Second language acquisition process of learners is a process that gradually gets rid of these limits of speech cognitive processing strategies.[1][2] The study on the speech processing strategies went deep into the learners' speech processing mechanism, which was more thorough and owned higher theoretical values. Second language acquisition studies of multiple languages have verified the scientificity and rationality of this theory.[3][4][5][6][7]

Some scholars [8][9][10] have made studies on second language acquisition of Chinese Bi-sentences. However, these studies mostly focus on error analysis and acquisition order and they fail to study the speech processing strategies. Zhu Yunfeng[11] studied the speech processing strategies of Chinese comparative-sentences of Korean foreign students, but due to limits of conditions, her research scope was oversized and the research process was not rigorous. Wang Ruifeng[12]made a comparative study on the speech processing strategies of Bi-sentences of English native speakers and Japanese native speakers. It was found that though English native speakers and Japanese native speakers had different native languages, they utilized basically consistent speech processing strategies in the acquisition of 9 patterns of Chinese

This research was financially supported by Science Foundation of BLCU( Supported by "the Fundamental Research Funds for the Central Universities”),13YBG09.
Bi-sentences and their development process of the speech processing strategies also basically coincided. Thus, it was deduced that the speech processing strategies might be a common and universal cognitive and processing method. Wang Ruifeng came to the above conclusion only according to the comparison between English native speakers and Japanese native speakers in the speech processing strategies of $\mathrm{Bi}$ sentences. Could it apply to other conditions? Also, could these speech processing strategies be applied to other language native speakers? Following will verify it by investigating the acquisition conditions of Bi-sentences of Thai native speakers with the same experimental materials and procedures.

\section{RESEARCH DESIGN}

The basic idea and method of our study are as follows.

Interlanguage corpus of 9 patterns of Chinese Bi-sentences of intermediate Thai native speakers is collected by test paper (specific to 9 patterns of Chinese Bi-sentences).

According to the obtained interlanguage corpus, the acquisition characteristics and acquisition stages of 9 patterns of Chinese Bi-sentences of intermediate Thai native speakers are investigated.

According to the acquisition characteristics and acquisition stages of 9 patterns of Chinese Bi-sentences, the speech processing strategies of Chinese $\mathrm{Bi}$-sentences of intermediate Thai native speakers are explored.

\section{ACQUISITION STAGES OF CHINESE BI-SENTENCES OF THAI NATIVE SPEAKERS}

A. Implication Scale and Coefficient of Chinese Bi-sentences of Intermediate Thai Native Speakers

According to the implication scale of various comparativesentences of intermediate Thai native speakers, indicator coefficients are calculated.

Coefficient of Reproducibility (Crep): 0.96

Minimum Marginal Reproducibility (MMrep): 0.82

Percent Improvement in Reproducibility: 14\%

Coefficient of Scalability (Cscal): 0.78

It can be seen from the above indicator coefficients that the coefficient of scalability in the implication scale of various 
comparative-sentences of intermediate Thai native speakers is 0.78 , which is higher than the critical value, 0.60 . This indicates that this implication scale manifests a strong implication relation and can be taken as the prediction basis of the acquisition of various comparative-sentences of intermediate Thai native speakers.

\section{B. Cluster Analysis Results of Chinese Bi-sentences of Intermediate Thai Native Speakers}

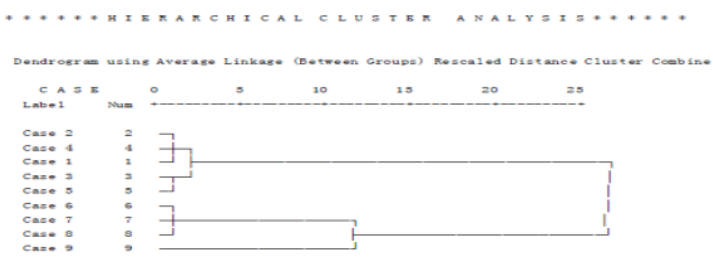

Fig. 1. Tree diagram of two-list variable cluster

It can be seen from tree diagram of two-list variable cluster analysis that the distances between case 1, case 2 and case 4 are small and they can be clustered into one type. Case 3 and case 5 are clustered into one type. The distances between case 6, case 7 and case 8 are small and they can be clustered into one type.

\section{Acquisition Stage of Chinese Bi-sentences of Intermediate} Thai Native Speakers

According to the cluster analysis results of 9 patterns of Chinese Bi-sentences of intermediate Thai native speakers, the development stage of the acquisition process of intermediate Thai native speakers can be roughly drawn.

TABLE I. ACQUISITION STAGE OF CHINESE BI-SENTENCES OF INTERMEDIATE THAI NATIVE SPEAKERS

\begin{tabular}{|c|c|}
\hline Acquisition stage & case \\
\hline \multirow[t]{3}{*}{ First stage } & case 1 \\
\hline & case 2 \\
\hline & case 4 \\
\hline \multirow[t]{2}{*}{ Second stage } & case 3 \\
\hline & case 5 \\
\hline \multirow[t]{3}{*}{ Third stage } & case 8 \\
\hline & case 6 \\
\hline & case 7 \\
\hline Forth stage & case 9 \\
\hline
\end{tabular}

The acquisition stages classified by the cluster analysis roughly reflect the acquisition order of 9 patterns of Chinese Bi-sentences of intermediate Thai native speakers, namely, the order from top to bottom in the above table.

\section{The Speech Processing Strategies of BI- SENTENCES OF THAI NATIVE SPEAKERS}

\section{A. The Speech Processing Strategies for Word Order of Bi- sentences of Thai Native Speakers}

From word order, the word order processing of inner components of Bi-sentences of Thai native speakers goes through the dominance of two word order strategies from bottom to top.
First is the word order strategy for single typical Bisentences. 9 patterns of Chinese Bi-sentences mainly can be classified into two types. One is basic sentence patterns without difference quantity and the other one is extension sentence patterns with difference quantity. Most patterns of Chinese Bisentences with difference quantity are generated by adding difference quantity to relevant basic sentence patterns. For example, case 2, case 3 and case 4 are generated by adding the difference quantity to the comparative difference of basic case 1. Case 6 and case 7 are extended by adding difference quantity to the comparative difference of case 5 . This order can be seen a typical word order of Bi-sentences. However, case 9 is an exception. It not only is from adding the difference quantity to the comparative difference of basic case 5 and meanwhile adding the difference quantity at the end, but also places the comparative difference (earlier, later, more and less) ahead of verbs. Compared with word orders of other sentences with the difference quantity, the word order of case 9 is a special nontypical Bi-sentence word order.

The test results indicate that most testees of Thai native speakers use the word order strategy for typical Bi-sentences to process 9 patterns of Chinese Bi-sentences. All Thai native speakers all acquire case 2 and case 4 and nearly 90\% Thai native speakers acquire case 3 . Though learners have made a certain number of errors in case 3, for example, A Bi B + You Dian Er + adjective, these errors are regarded as component misuse bias errors, instead of word order bias errors. This problem will be analyzed below.

Less than half Thai native speakers acquire case 6, with 12 testees, accounting for $40 \%$ of all testees. These Thai native speakers have acquired the word order of this case. Bias error of $\mathrm{A} \mathrm{Bi} \mathrm{B} \mathrm{+} \mathrm{verb} \mathrm{+} \mathrm{adjective} \mathrm{+} \mathrm{You} \mathrm{Dian} \mathrm{Er} \mathrm{is} \mathrm{observed} \mathrm{of}$ Thai native speakers. They belong to bias error, but they conform to typical Bi-sentence word order of comparative subject + comparative word + comparative object + comparative difference + difference quantity. If these bias errors are calculated, the number of Thai native speakers acquiring this word order will increase a lot. There are a certain number of other types of bias errors in case 6 , for example, $\mathrm{A}$ $\mathrm{Bi}$ B + verb + You Dian Er + adjective, A Bi B + verb + De + You Dian Er + adjective. However, as the analysis of case 3, these bias errors are regarded as bias error in component misuses, instead of bias error in word order.

For case 7, less than half Thai native speakers acquire it, with 11 testees, accounting for $37 \%$ of all testees. These Thai native speakers have acquired the word order of this case. Bias error of A Bi B + verb + adjective + Deduo are observed a lot among Thai native speakers. They conform to typical Bisentence word order of comparative subject + comparative word + comparative object + comparative difference + difference quantity. If these bias errors are calculated, the number of Thai native speakers acquiring this word order will increase a lot. There are a certain number of other types of bias errors for case 7, for example, A Bi B + verb + De + adverb of degree + adjective, $\mathrm{A} \mathrm{Bi} \mathrm{B}+$ verb + adverb of degree + adjective. However, as the analysis of case 4, these bias errors are regarded as bias error in component misuses, instead of bias error in word order. 
From the above acquisition condition of sentences, it can be seen that most learners utilize typical Bi-sentence word order to produce the above various $\mathrm{Bi}$-sentences.

Thai native speakers also apply this rule to typical Bisentence word order of case 9. Even numerous teaching and drills are made on case 9, but Thai native speakers still obtain poor acquisition. Among bias errors of case 9, many belong to this kind of typical Bi-sentence word order. For example, two types of bias errors occur most, A Bi B + verb + ealier/ later/ more/ less + degree-quantity and $\mathrm{A} \mathrm{Bi} \mathrm{B} \mathrm{+} \mathrm{verb} \mathrm{+} \mathrm{+} \mathrm{De} \mathrm{+}$ earlier/ later/ more/ less. Actually they are from the basic sentence pattern, A Bi B + verb + (De) + earlier/ later/ more/ less as well degree-quantity (difference quantity). This belongs to typical Bi-sentence word order. Both the number of bias errors and the testees with bias errors account for extremely high proportions in bias errors of this sentence pattern.

Only 3 Thai native speakers acquire case 9, who successfully skips the dominance of the word order strategy for single typical Bi-sentences and they realize how to adopt the corresponding Bi-sentence word order under different conditions. They not only adopt the strategy for typical Bisentences in typical Bi-sentence word orders but also adopt the strategy for non-typical Bi-sentences in non-typical Bisentence word orders. Namely, they can use corresponding multi-conditional word order strategy under different conditions, instead of adopting single word order strategy to cognize all Bi-sentences. Their cognitive strategies have reached the word order strategy for multi-conditional $\mathrm{Bi}$ sentences.

According to the above analysis, Thai native speakers go through the development process from the word order strategy for single typical Bi-sentences to the word order strategy for multi-conditional Bi-sentences.

The above two word order strategies can be shown as two stages. Thai native speakers need to gradually span these two word order strategies from bottom to top in the acquisition of the word order of Bi-sentences. First of all, Thai native speakers board the first stage. Comparatively speaking, the first stage is easy to surpass. Almost all Thai native speakers can easily and correctly use this strategy. Then, Thai native speakers board the second stage. It is very difficult for them to surpass the second stage. Only a few Thai native speakers can board this stage.

\section{B. The Speech Processing Strategies of Inner-Sentence Semantic Components of Thai Native Speakers}

It can be seen from inner-sentence semantic components that many Thai native speakers is dominated by the strategy of the single typical word combination, namely, many Thai native speakers combine semantic components of Bi-sentences according to some learnt typical word combination rules.

For case 3 and case 6, many Thai native speakers make bias errors of You Dian Er. Both the number of bias errors and the testees with bias errors account for certain proportions in the bias errors of these two cases. Before learning these two $\mathrm{Bi}$ sentences, testees have learnt Yi Dian Er and You Dian Er. Their basic difference lies in that Yi Dian Er is placed ahead of noun while You Dian Er is placed ahead of adjective. For example, You Dian Er Pang and You Dian Er Kuai. Also, numerous drills are made. The word combination rule of You Dian Er is seen as typical word combination rule and this is a Chinese typical expression rule of degree small quantity. However, in Bi-sentences, Yi Dian Er is used and placed after adjective, belonging to a typical word combination rule. It can be seen that when acquiring Bi-sentences, Thai native speakers still utilize the typical word combination rule of You Dian Er, producing bias errors of A Bi B + You Dian Er + adjective, A $\mathrm{Bi} \mathrm{B}+$ verb + You Dian Er + adjective, and A Bi B + verb + $\mathrm{De}+$ You Dian Er + adjective.

For use of case 4 and case 7, many Thai native speakers also are dominated by typical word combination rules and make many bias errors of adverb of degree. Before learning these two cases, they have learnt the general rule of Chinese degree of Da, namely, adverb of degree (Hen, Fei Chang et al.,) is placed ahead of adjective, for example, Fei Chang Shou and Hen Jin. This kind of word combination rule of degree of Da is a typical word combination rule and also is a typical expression rule of degree of Da. However, in Bi-sentences, this typical rule is not used. Instead, adjective + De Duo is used to express degree of Da, which is a non-typical expression rule. It can be seen that when acquiring Bi-sentences, many Thai native speakers still adopt the typical word combination rule that adverb of degree is placed ahead of adjective, producing bias errors of $\mathrm{A} \mathrm{Bi} \mathrm{B} \mathrm{+} \mathrm{verb} \mathrm{+} \mathrm{adverb} \mathrm{of} \mathrm{degree} \mathrm{+} \mathrm{adjective,} \mathrm{and} \mathrm{A}$ $\mathrm{Bi} \mathrm{B}+\mathrm{verb}+\mathrm{De}+$ adverb of degree + adjective.

From the test results, it can be seen that of Thai native speakers at this level, some have mastered these four cases, respectively with 25, 30, 12 and 11 of case 3, case 4, case 6 and case 7. If only the correct use of these semantic components in Bi-sentences is paid attention to, Thai native speakers with bias errors of A Bi B + verb + adjective + Yi Dian Er and A Bi B + verb + adjective + De Duo also can be considered to have acquired non-typical word combination rule. In this way, the number of testees grasping non-typical word combination rules also increases. The testees who can correctly use non-typical word combination rules and those who have acquired these cases can employ corresponding rules to combine words in $\mathrm{Bi}$ sentences with non-typical word combination rules. Their cognitive strategies are improved to the strategy of multiconditional word combination.

According to the above analysis, Thai native speakers go through the development process from the strategy of the single typical word combination to the strategy of multi-conditional word combination from inner-sentence semantic components.

\section{The Speech Processing Strategies of Inner-Sentence Grammatical Component of Thai Native Speakers}

It can be seen from inner-sentence semantic components that many Thai native speakers is dominated by the strategy of skipping grammatical components, namely, many Thai native speakers skip grammatical component, De, after verb, which has no semantic meaning and plays a grammatical role to express the modifying relationship between verb and complement. The skipping of this grammatical component has no influence on the semantic expression and it interferes the 
normal communication of information little. To certain degree, De is insignificant redundant information in semantic expression and testees tend to ignore this kind of component and skip it in expression. VanPatten (2007) also referred to this phenomenon in his discussion of linguistic form processing in the second language input understanding. Namely, the working memory capacity of learners was limited and to understand sentences and acquire the meaning of sentences, they firstly focused on notional words, instead of grammatical form. When notional words and grammatical form expressed the same grammatical meaning, the grammatical meaning expressed by grammatical form was redundant information. Learners usually adopt the processing strategy of notional words first and grammatical meaning later.

For uses of case 5, case 6, case 7 and case 8, many testees skip grammatical component, De, generating bias errors of $\mathrm{A}$ $\mathrm{Bi} \mathrm{B}+$ verb + adjective, $\mathrm{A} \mathrm{Bi} \mathrm{B}+$ verb + adjective + Yi Dian Er, A Bi B + verb + adjective + De Duo. Thus, it can be seen that many learners usually adopt the strategy of skipping grammatical components without any semantics, paying attention to semantic expression while ignoring grammatical components.

It can be seen from test results that some testees have acquired these four cases, respectively with 25, 12, 11 and 14 of case 5 , case 6 , case 7 and case 8 . It is found that bias errors of many testees contain De while other components of sentences are found wrong, for example, A Bi B + verb + De + Yi Dian Er + adjective, A Bi B + verb + De + adverb of degree + adjective, and $\mathrm{A} \mathrm{Bi} \mathrm{B} \mathrm{+} \mathrm{verb} \mathrm{+} \mathrm{object}+$ De + adjective. If only focusing on the use of grammatical component, De and ignoring the correct or false use of other components, testees with these bias errors actually grasp this grammatical component, De. For the testees who use De and those who have acquired these cases all pay attention to this grammatical component and utilize it. Their cognitive strategies are improved to the strategy of using grammatical component.

According to the above analysis, Thai native speakers go through the development process from the strategy of skipping grammatical components to the strategy of using grammatical components.

\section{CONCLUSION}

Through the above analysis, it can be seen that Thai native speakers go through the common spanning process of the speech processing strategies in terms of the development process of the speech processing strategies. Its speech processing strategies for word order experience the development process from the word order strategy for single typical Bi-sentences to the word order strategy for multiconditional Bi-sentences. The speech processing strategies for inner-sentence semantic components go through the development process from the strategy of the single typical word combination to the strategy of multi-conditional word combination. The speech processing strategies for innersentence grammatical components experience the development process from the strategy of skipping grammatical components to the strategy of using grammatical components.

\section{DISCUSSION}

Thai native speakers differ a little from English native speakers and Japanese native speakers in the speech processing strategies of Bi-sentence word order, namely, Thai native speakers do not go through the stage of the word order strategy for simple Bi-sentences and they start from the word order strategy for single typical Bi-sentences. They make no bias error in case 3. However, English native speakers and Japanese native speakers make the bias error of $\mathrm{A} \mathrm{Bi} \mathrm{B} \mathrm{+} \mathrm{complement}+$ precision measure + adjective. Why is there no such a bias error of Thai native speakers in case 3? It is found from the analysis of the corresponding sentence patterns of English, Japanese and Chinese that the difference quantities of the corresponding sentence patterns of English and Japanese is ahead of difference. The location of the difference quantity of the corresponding sentence patterns of Thai is the same as Chinese, behind difference. This may be the reason for no bias errors in case 3 of Thai native speakers, namely, the consistency between Thai and Chinese plays an actively positive transfer in the acquisition of Thai native speakers. It is a pity that this paper only studies the acquisition condition of intermediate Thai native speakers, and there is no investigation in the acquisition condition of primary Thai native speakers. If primary Thai native speakers also have no bias errors in case 3, it can verify the existence and role of positive transfer more clearly. The future study will make up for this shortcoming.

\section{REFERENCES}

[1] J. Meisel, H. Clashsen and M. Pienemann, “On determining developmental stages in natural second language acquisition”, Studies in Second Language Acquisition, vol. 2, pp. 109-353, 1981.

[2] M. Pienemann, "Psychological constraints on the teachability of languages”, Studies in Second Language Acquisition, vol. 2, pp. 186214, 1984.

[3] M. Pienemann, M. Johnston and G. Brindley, "Constructing an acquisition-based procedure for assessing second language acquisition”, Studies in Second Language Acquisition, vol. 2, pp. 217-243, 1988.

[4] A. Mackey and J. Philp, "Conversational interaction and second language development: Recasts, responses and red herrings?”, The Modern Language Journal, vol. 3, pp. 338-356, 1998.

[5] K. Yoshioka and T. Doi, "Testing the Pienemann-Johnston model with Japanese: a speech-processing view of the acquisition of particles and word order”, Paper presented at the 8th Second Language Research Forum, Hawaii: University of Hawaii, 1988.

[6] Sun Shuzi, Study on the Speech Processing Strategies of the Acquisition of Chinese Disyllables VO-Type Detachable Words, Master dissertation of Beijing Language and Culture University, 2004.

[7] Li Jiancheng, Study On The Speech Processing Strategies in the Acquisition Process of Chinese Directional Complements of Korean Foreign Students, Master dissertation of Beijing Language and Culture University, 2007.

[8] Xiao Xiaoping, An Analysis on the Errors and an Inspection on the Acquisition Order of Chinese Comparative-Sentences by Vietnamese Oversea Students, Master dissertation of Guangxi Normal University, 2004.

[9] Chen Jun, Zhou Xiaobing, The Selection and Arrangement of Grammatical Items Concerning Comparative Sentences, Language Teaching and Linguistic Studies, vol. 2, 2005.

[10] Wang Maolin, A Study on Foreign Learners' Acquisition of Bi-Structure. Journal of Jinan University College of Chinese Language and Culture, vol. 3, 2005. 
[11] Zhun Yunfeng, Study on Acquisition Order Inspection and the Speech Processing Strategies of Chinese Comparative Sentences of Korean Students, Master dissertation of Beijing Language and Culture University, 2008.

[12] Wang Ruifeng , Comparative Study on the Speech Processing Strategies of Bi-Sentences of English Native Speakers and Japanese Native Speakers, Beijing: Beijing Language and Culture University Press, 2014. 